\title{
Balloon sizing for measuring patent ductus arteriosus size in transcatheter closure using amplatzer duct occluder is simple and accurate: Report of 3 cases
}

\author{
Chaerul Achmad $^{1 *}$, Toni Mustahsani Aprami ${ }^{1}$, Augustine Purnomowati ${ }^{1}$, Endah Sri Rahayuningsih ${ }^{1}$, Achmad Fauzi Yahya ${ }^{1}$ and Muhammad \\ Munawar $^{2}$ \\ ${ }^{1}$ Faculty of Medicine, Univeristas Padjadjaran, Hasan Sadikin General Hospital, Bandung, Indonesia \\ ${ }^{2}$ Bina Waluya Cardiac Centre, Jakarta, Indonesia
}

\begin{abstract}
Transcatheter closure is the preferred method of treatment of patent ductus arteriosus. Angiographic measurement of patent ductus arteriosus size is now widely used, but this technique needs more contrast and more importantly in some selected cases, the size of the patent ductus arteriosus cannot be seen clearly. Therefore, we report our series of using balloon sizing for measuring patent ductus arteriosus size in patent ductus arteriosus closure procedure. We hypothesize that there are some advantages of using this technique. It will reduce the contrast material, easier to delineating the size, and more importantly it is more accurate sizing because of having a larger its reference ( $15 \mathrm{~mm}$ for balloon versus $2 \mathrm{~mm}$ for $6 \mathrm{~F}$ catheter size in angiographic technique).

We report our 3 series of isolated patent ductus arteriosus cases. All were female, and their age ranged 16 to 30 year-old. The patent ductus arteriosus was easily measured using $24 \mathrm{~mm}$ sizing balloon (AGA Medical Inc, USA) by pulling back from the aorta to pulmonary artery. The sizes of each patent ductus arteriosus were $8.4 \mathrm{~mm}, 8.7 \mathrm{~mm}$, and $6.3 \mathrm{~mm}$ respectively. All could be accurately and successfully closed with 12/10, 12/10 and 10/8 mm of amplatzer duct occluder. The contrast needed was only $30 \mathrm{cc}$ for each individual for evaluating post-amplatzer duct occluder outcome.
\end{abstract}

Conclusion: Balloon sizing for measuring patent ductus arteriosus size in transcatheter closure is simple and accurate and need less contrast.

\section{Introduction}

Patent ductus arteriosus is the persistence opening structure between the left pulmonary artery and the descending aorta. Persistence of this fetal structure beyond 10 days of life is considered abnormal. Left-to-right shunting through the ductus arteriosus results in pulmonary over circulation and left heart volume overload. However, patients are exposed to increased risk of infective endocarditis. Open surgical treatment of patent ductus arteriosus in adults is technically more difficult than in children. Transcatheter closure of the ductus in adults has become an attractive alternative to surgery. Patent ductus arteriosus device closure was first attempted by porstmann et al in 1967 [1]. However, complications of transcatheter closure of patent ductus arteriosus include residual shunt, left pulmonary artery obstruction, protrusion of the device into the aorta, so the exact measurement of patent ductus arteriosus size is the most important in patent ductus arteriosus occlusion by transcatheter [2,3]. These are 3 cases reports of patent ductus arteriosus closure which using the balloon measurement.

\section{Cases}

3 patients female with their age range 16 to 30 year-old underwent transcatheter closure of patent ductus arteriosus. All the patients were admitted one day before the procedure for clinical, laboratory and echocardiographic assessment. The procedures were carried out via the right femoral approach under local anaesthesia. The presence of a patent ductus arteriosus and was confirmed by lateral cineangiogram in the descending aorta. Measurement of patent ductus arteriosus size was done by pulling back $24 \mathrm{~mm}$ sizing balloon (AGA medical Inc,
USA) from the aorta to pulmonary artery. The size of patent ductus arteriosus was calculated. Diameter of patent ductus arteriosus divided by reference of sizing balloon times $15 \mathrm{~mm}$. Descending aortogram was performed after the Amplatzer duct occluder device implantation to check for residual patent ductus arteriosus flow. The usage of contrast was $30 \mathrm{cc}$ when aortogram done (Figures 1, 2 and 3).

\section{Discussion}

Patent ductus arteriosus, naturally, is asymptomatic, so detection during childhood commonly undetectable. However, increased pulmonary flow from the ductal shunting leads to increased pulmonary fluid volume and prolong shunting can result silent pulmonary hypertension and left ventricular dysfunction. Unfortunately, open surgical treatment of patent ductus arteriosus in adults is technically more difficult than in children. With the increasing of percutaneous approach, patients started to undergo this minimally invasive method of patent ductus arteriosus occlusion. Transcatheter closure of patent ductus arteriosus is a safe and effective alternative therapeutic modality to surgical intervention, although the complication can occur during or

Correspondence to: Chaerul Achmad, Cardiology and Vascular Medicine Department, Hasan Sadikin General Hospital, Jalan Pasteur no 38, Bandung, Indonesia, Tel: +6282218071997; E-mail: Chaerula2015@yahoo.com

Key words: patent ductus arteriosus, amplatzer duct occluder, contrast

Received: November 05, 2017; Accepted: November 23, 2017; Published: November 27, 2017 
Achmad C (2017) Balloon sizing for measuring patent ductus arteriosus size in transcatheter closure using amplatzer duct occluder is simple and accurate: Report of 3 cases

after intervention. One of the complications of patent ductus arteriosus closure is residual shunt. So, measurement of diameter patent ductus arteriosus is the most important technique before choosing amplatzer duct occluder size. The bigger size as reference of patent ductus arteriosus measurement the more accurate in patent ductus arteriosus occlusion. In our cases, we use $24 \mathrm{~mm}$ sizing balloon (AGA Medical Inc, USA) by pulling back from the aorta to pulmonary artery. In the past, the reference was using $2 \mathrm{~mm}$ of $6 \mathrm{~F}$ catheter during angiographic technique. The sizes of each patent ductus arteriosus in our patients were $8.4,8.7$ and $6.3 \mathrm{~mm}$ respectively. all could be accurately and successfully closed with $12 / 10,12 / 10$ and $10 / 8 \mathrm{~mm}$ of amplatzer duct occluder (amplatzer duct occluder). the contrast needed was only $30 \mathrm{cc}$ for each individual for evaluating post-amplatzer duct occluder outcome.

\section{Conclusion}

Balloon sizing for measuring patent ductus arteriosus size in transcatheter closure is simple and accurate and need less contrast.
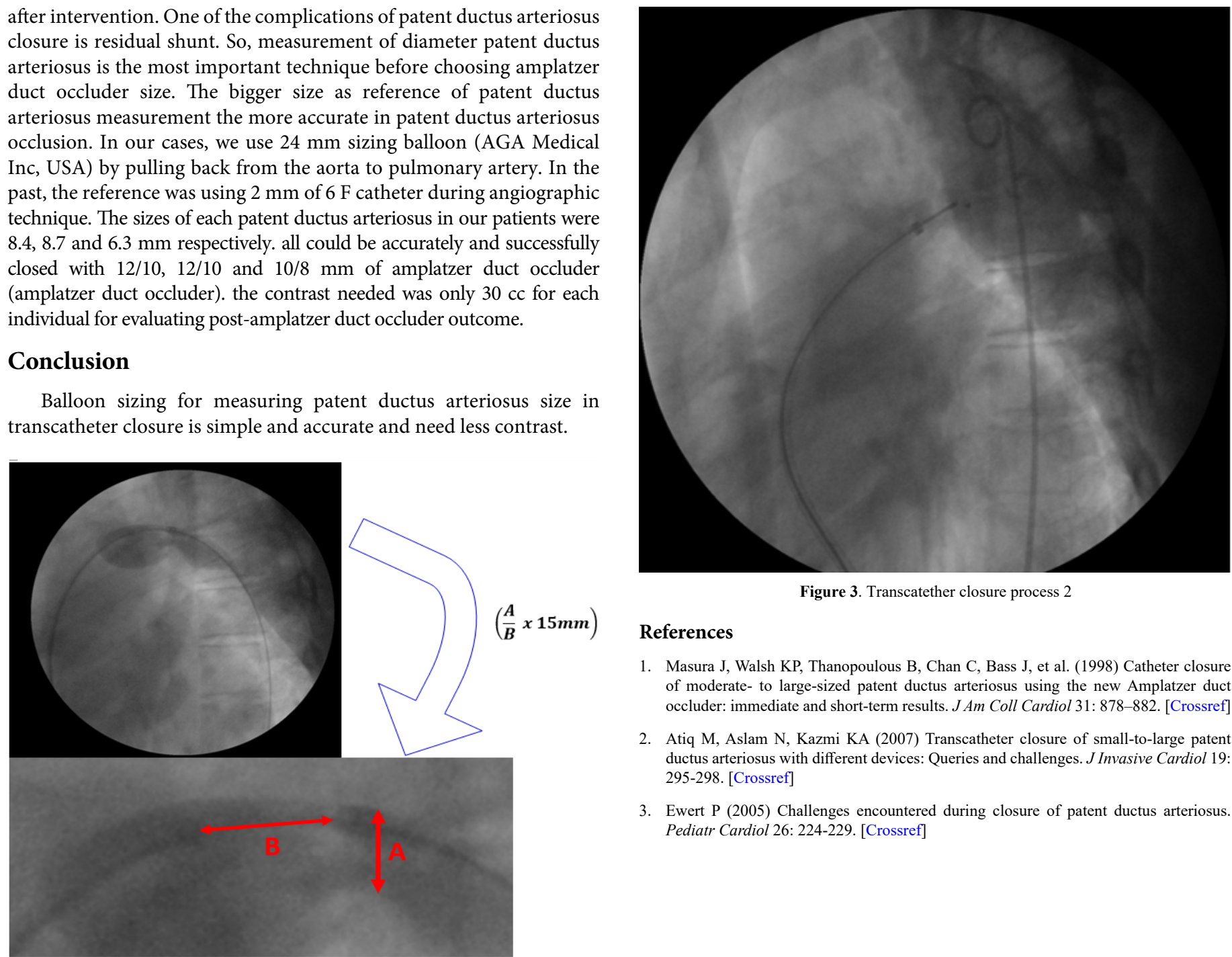

Figure 3. Transcatether closure process 2

\section{References}

1. Masura J, Walsh KP, Thanopoulous B, Chan C, Bass J, et al. (1998) Catheter closure of moderate- to large-sized patent ductus arteriosus using the new Amplatzer duct occluder: immediate and short-term results. J Am Coll Cardiol 31: 878-882. [Crossref]

2. Atiq M, Aslam N, Kazmi KA (2007) Transcatheter closure of small-to-large patent ductus arteriosus with different devices: Queries and challenges. J Invasive Cardiol 19: 295-298. [Crossref]

3. Ewert P (2005) Challenges encountered during closure of patent ductus arteriosus Pediatr Cardiol 26: 224-229. [Crossref]

Figure 1. EKG Clinical Ventricular Tachycardia

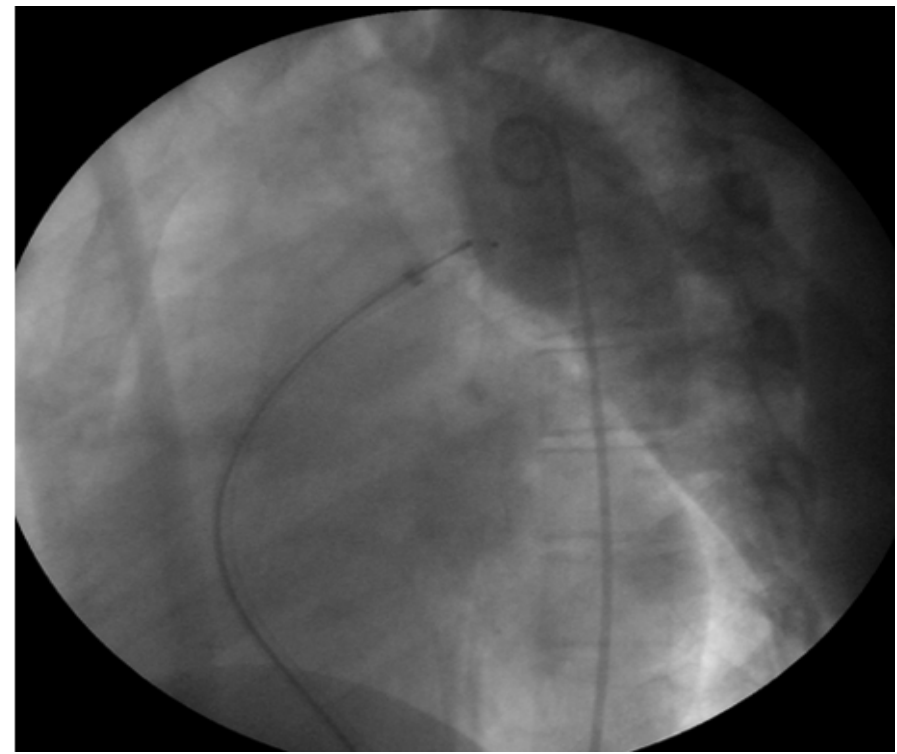

Figure 2. Transcatether closure process 1
Copyright: (C)2017 Achmad C. This is an open-access article distributed under the terms of the Creative Commons Attribution License, which permits unrestricted use, distribution, and reproduction in any medium, provided the original author and source are credited. 